\title{
Effects of hypercapnia in sepsis: protocol for a systematic review of clinical and preclinical data
}

\author{
Thomas P. Clyde ${ }^{1}$, Michael Coletta ${ }^{1}$, Christopher Jones ${ }^{1}$, Hope Kilgannon' ${ }^{1}$ Brian M. Fuller ${ }^{3}$, Stephen Trzeciak ${ }^{1,2}$
} and Brian W. Roberts ${ }^{* *}$

\begin{abstract}
Background: Respiratory failure requiring mechanical ventilation is a common manifestation of end-organ damage among patients with sepsis and has a high morbidity and mortality rate, as well as substantial associated treatment costs. Considering the burden of this condition, there is great need to identify novel, pragmatic therapies to improve outcomes in this population. Hypercapnia has shown benefits in several different ex vivo and in vivo models of lung injury. However, it is currently unclear if hypercapnia can confer clinical benefit among patients with sepsis. The objective of this systematic review is to collate the biomedical literature of preclinical and clinical studies testing the effects of higher $\mathrm{PaCO}_{2}$ levels in the setting of sepsis.
\end{abstract}

Methods: We will perform a qualitative systematic review of preclinical and clinical studies evaluating the effects of hypercapnia in sepsis. We will search CENTRAL, PubMed, CINAHL, and EMBASE using a comprehensive strategy. We will screen the reference lists of the articles we select for inclusion to identify additional studies for potential inclusion. Two independent reviewers will review all search results. Upon inclusion of articles, we will extract data using a standardized form. We will use tables to describe the study type, population included, exposure and control groups, outcome measures, and effects of exposure on outcome measures compared to controls.

Discussion: This systematic review aims to synthesize the world's literature on the effects of hypercapnia in the setting of sepsis. We expect this systematic review will find that majority of the studies will demonstrate a potential benefit of higher $\mathrm{PaCO}_{2}$ levels in sepsis. The results of this systematic review will contribute to the understanding of the effects of hypercapnia in the setting of sepsis and promote future research of $\mathrm{PaCO}_{2}$ management in mechanically ventilated patients with sepsis.

Systematic review registration: The systematic review is registered in the PROSPERO international prospective register of systematic review (PROSPERO \# CRD42018086703).

Keywords: Hypercapnia, Hypoventilation, Mechanical ventilation, Sepsis, Septic shock, Respiratory failure, Review

\section{Background}

Sepsis places an enormous burden on healthcare systems across the globe due to its high incidence, substantial costs of treatment, and high mortality rate [1]. Patients with septic shock often develop respiratory failure, with as many as $80 \%$ requiring mechanical ventilation [2].

\footnotetext{
* Correspondence: roberts-brian-w@cooperhealth.edu

${ }^{1}$ The Department of Emergency Medicine, Cooper University Hospital, Cooper Medical School of Rowan University, One Cooper Plaza, K152, Camden, NJ 08103, USA

Full list of author information is available at the end of the article
}

Sepsis patients requiring mechanical ventilation are at high risk for ventilator-associated complications [3, 4] and have a mortality rate approaching $50 \%[2,5]$. Considering the high incidence and mortality rate associated with mechanical ventilation among patients with sepsis, there is a need to identify novel and pragmatic approaches to improve outcomes in patients with sepsis-associated respiratory failure.

The management of the partial pressure of arterial carbon dioxide $\left(\mathrm{PaCO}_{2}\right)$ is a fundamental aspect of care in all mechanically ventilated patients. The conventional

(c) The Author(s). 2018 Open Access This article is distributed under the terms of the Creative Commons Attribution 4.0 International License (http://creativecommons.org/licenses/by/4.0/), which permits unrestricted use, distribution, and 
paradigm dictates that the mechanical ventilator should be adjusted to target a normal physiological $\mathrm{PaCO}_{2}$ range. However, data has shown that elevated $\mathrm{PaCO}_{2}$ in the setting of a lung-protective ventilation strategy (i.e., permissive hypercapnia) is well tolerated and may be associated with improved survival among mechanically ventilated patients with sepsis [6]. In addition to the advantages of low stretch ventilation, preclinical data suggests hypercapnia may protect against systemic organ injury through attenuation of inflammation and free radical generation $[7,8]$. The ubiquitous need to manage $\mathrm{PaCO}_{2}$, combined with data suggesting benefit associated with hypercapnia, suggests that $\mathrm{PaCO}_{2}$ could be a target to improve outcomes in this vulnerable patient population.

The aim of this systematic review is to collate the biomedical literature of (1) preclinical studies testing the effects of higher $\mathrm{PaCO}_{2}$ levels in the setting of sepsis and (2) clinical investigations testing the effects of hypercapnia on clinical outcomes in mechanically ventilated patients with sepsis. Our overarching hypothesis is that the majority of the studies will demonstrate a potential benefit of higher $\mathrm{PaCO}_{2}$ levels in sepsis. If the preponderance of data suggests hypercapnia attenuates sepsis-induced injury, then this report will provide scientific rationale for implementing high-quality interventional trials to test the effects of targeting elevated $\mathrm{PaCO}_{2}$ levels in mechanically ventilated patients with sepsis.

\section{Methods/design}

\section{Protocol and registration}

This systematic review protocol was prepared according to the guidelines set forth in the Cochrane Handbook for the Systematic Reviews of Interventions [9] and Preferred Reporting Items for Systematic Review and Meta-Analysis Protocols (PRISMA-P) 2015 (Supplemental Material) [10]. This protocol's PROSPERO registration number is CRD42018086703.

\section{Search for and identification of studies}

We will search CENTRAL, PubMed, CINAHL, and EMBASE using the following search terms: "hypoventilation" or "hypercapnia" or "hypercarbia" or "carbon dioxide" or "CO2" AND "bacterial infection" or "pneumonia" or "sepsis" or "septic shock" or "bacteremia." We modeled our search terms after search terms used in previously published systematic reviews $[11,12]$. We will also screen reference lists of the articles we select for inclusion to identify additional studies for potential inclusion.

\section{Eligibility criteria}

We will include all study designs of the effects of hypercapnia in sepsis, severe sepsis, or septic shock. If a study population does not meet the inclusion criteria but a clearly defined subset of patients meets the inclusion criteria, the study may be included; however, only data pertaining to that subset of patients will be collected. The inclusion criteria for preclinical studies are as follows: (1) sepsis model (e.g., cecal ligation), (2) documented measurement of $\mathrm{PaCO}_{2}$, and (3) comparison of outcome measure between different $\mathrm{PaCO}_{2}$ levels. The inclusion criteria for clinical studies are as follows: (1) patients diagnosed with sepsis (including severe sepsis and septic shock; given we will be including paper regardless of date of publication and the variation in sepsis definitions over time, we will include any papers in which patients are diagnosed with an infection and have some definition of systemic involvement), (2) patients receiving invasive mechanical ventilation, (3) documented measurement of $\mathrm{PaCO}_{2}$, and (4) comparison of outcomes between different $\mathrm{PaCO}_{2}$ levels. We will consider studies eligible for review regardless of study design, language, or publication type. We will exclude studies that are secondary reports of studies already included. We also will exclude papers that are reviews, correspondence, or editorials; however, we will screen the reference lists of review articles to identify further studies for inclusion.

\section{Study selection and data abstraction}

Two independent reviewers will screen the titles and abstracts of identified studies for potential eligibility. After the relevance screen, the two reviewers will compare their exclusion logs and use the kappa statistic to quantify the inter-observer agreement. In cases of disagreement, the full text will be reviewed for inclusion. All studies deemed potentially relevant will be obtained and the full manuscripts will be reviewed for inclusion. Two reviewers will independently abstract data on all patient populations, interventions, outcome measures, adverse events, and results using a standardized data collection form.

\section{Assessment of study bias}

We will follow the Cochrane Handbook's recommendations for assessing risk of bias in clinical and non-clinical trials [9]. For clinical trials, we will use the Cochrane Collaboration's tool which evaluates risk of bias in six domains: selection, performance, detection, attrition, reporting, and other biases [9]. For observational human studies, we will use the New Castle-Ottawa Quality Assessment scale [9]. For animal models, we will use the Systematic Review Centre for Laboratory Animal Experimentation's (SYRCLE) review of bias tool [13]. The overall strength of the body of evidence will be assessed using the Grading of Recommendations Assessment, Development and Evaluation (GRADE) [14]. 


\section{Analysis}

We will perform a primarily qualitative analysis of the data in accordance with the recommended methodology for qualitative reviews published in the Cochrane Handbook [9]. We will first divide manuscripts into two groups: (1) preclinical and (2) clinical.

For preclinical studies, we will table the following: (1) animal model (e.g., mouse, pig); (2) study design (e.g., randomized control trial, prospective cohort); (3) mechanism for inducing sepsis (e.g., endotoxin); (4) $\mathrm{PaCO}_{2}$ categories; (5) outcome measures, including primary and all secondary outcomes; (6) effects of hypercapnia on outcome measures; and (7) study quality (defined above).

For clinical studies, we will table the following: (1) study design (e.g., cohort, randomized clinical trial); (2) study population (i.e., study inclusion criteria, age, and sepsis definition used); (3) $\mathrm{PaCO}_{2}$ categories; (4) outcome measures, including primary and all secondary outcomes; (5) effects of hypercapnia on outcome measures; and (6) study quality (defined above).

\section{Protocol amendments}

In the event of any changes to this protocol, each amendment will be stated, a date will be provided for each amendment, and the reasoning behind each amendment will be made known.

\section{Discussion}

Sepsis-associated respiratory failure is a common cause of morbidity and mortality, and its treatment requires an enormous amount of medical resources [1, 15]. New, cost-effective interventions are needed to address this disease process. Hypercapnia has been proposed to modulate gene expression, attenuate lung inflammation, and mitigate ventilator-associated lung injury, thereby potentially providing benefit to mechanically ventilated patients with sepsis [16-20]. To our knowledge, there is no systematic review of the world's literature on the effects of hypercapnia in the setting of sepsis.

This systematic review aims to synthesize the world's literature on the effects of hypercapnia in the setting of sepsis. We expect this systematic review will find that the majority of the studies will demonstrate a potential benefit of higher $\mathrm{PaCO}_{2}$ levels in sepsis. The results of this systematic review will contribute to the understanding of the effects of hypercapnia in the setting of sepsis and promote future research of $\mathrm{PaCO}_{2}$ management in mechanically ventilated patients with sepsis.

\footnotetext{
Abbreviations

GRADE: Grading of Recommendations Assessment, Development and Evaluation; $\mathrm{PaCO}_{2}$ : Partial pressure of arterial carbon dioxide; PRISMAP: Preferred Reporting Items for Systematic Review and Meta-Analysis Protocols; PROSPERO: International prospective register of systematic reviews; SYRCLE: Systematic Review Centre for Laboratory Animal Experimentation
}

Acknowledgements

None

\section{Funding}

This research received no specific grant from any funding agency in the public, commercial, or not-for-profit sector.

Availability of data and materials

This is a systematic review; all data are already publicly available.

\section{Authors' contributions}

All authors have made substantial contributions to this paper. BWR supervised all aspects of the study design and takes responsibility for the paper as a whole. All authors contributed to the development of the selection criteria, the risk of bias assessment strategy, and data extraction criteria. TPC, MC, and BWR developed the search strategy and drafted the manuscript. All authors read and contributed substantially to the revision of the final manuscript. All authors approved the manuscript in its final form.

Ethics approval and consent to participate

Not applicable

\section{Consent for publication}

Not applicable

\section{Competing interests}

The authors declare that they have no competing interests.

\section{Publisher's Note}

Springer Nature remains neutral with regard to jurisdictional claims in published maps and institutional affiliations.

\section{Author details}

${ }^{1}$ The Department of Emergency Medicine, Cooper University Hospital, Cooper Medical School of Rowan University, One Cooper Plaza, K152, Camden, NJ 08103, USA. ${ }^{2}$ The Department of Medicine, Division of Critical Care Medicine, Cooper University Hospital, Cooper Medical School of Rowan University, Camden, NJ, USA. ${ }^{3}$ Departments of Emergency Medicine and Anesthesiology, Division of Critical Care Medicine, Washington University School of Medicine, St. Louis, MO, USA.

Received: 16 April 2018 Accepted: 9 October 2018

Published online: 22 October 2018

\section{References}

1. Mayr FB, Yende S, Angus DC. Epidemiology of severe sepsis. Virulence. 2014; 5(1):4-11.

2. Quenot JP, Binquet $C$, Kara F, Martinet O, Ganster F, Navellou JC, Castelain V Barraud D, Cousson J, Louis G, et al. The epidemiology of septic shock in French intensive care units: the prospective multicenter cohort EPISS study. Crit Care. 2013;17(2):R65.

3. Dettmer MR, Mohr NM, Fuller BM. Sepsis-associated pulmonary complications in emergency department patients monitored with serial lactate: an observational cohort study. J Crit Care. 2015;30(6):1163-8.

4. Fuller BM, Mohr NM, Dettmer M, Kennedy S, Cullison K, Bavolek R, Rathert N, McCammon C. Mechanical ventilation and acute lung injury in emergency department patients with severe sepsis and septic shock: an observational study. Acad Emerg Med. 2013;20(7):659-69.

5. Mikkelsen ME, Shah CV, Meyer NJ, Gaieski DF, Lyon S, Miltiades AN, Goyal M, Fuchs BD, Bellamy SL, Christie JD. The epidemiology of acute respiratory distress syndrome in patients presenting to the emergency department with severe sepsis. Shock. 2013;40(5):375-81.

6. Roberts BW, Mohr NM, Ablordeppey E, Drewry AM, Ferguson IT, Trzeciak S, Kollef MH, Fuller BM. Association between partial pressure of arterial carbon dioxide and survival to hospital discharge among patients diagnosed with sepsis in the emergency department. Crit Care Med. 2018;46(3):e213-20.

7. Mehta AB, Syeda SN, Wiener RS, Walkey AJ. Epidemiological trends in invasive mechanical ventilation in the United States: a population-based study. J Crit Care. 2015;30(6):1217-21.

8. O'Croinin D, Ni Chonghaile M, Higgins B, Laffey JG. Bench-to-bedside review: permissive hypercapnia. Critical care (London, England). 2005;9(1):51-9. 
9. Higgins JPT, Green S: Cochrane Handbook for Systematic Reviews of Interventions Version 5.1.0 [updated March 2011]. The Cochrane Collaboration, 2011. Available from http://handbook-5-1.cochrane.org/. Accessed 21 Jan 2018.

10. Shamseer L, Moher D, Clarke M, Ghersi D, Liberati A, Petticrew M, Shekelle P, Stewart LA. Preferred reporting items for systematic review and metaanalysis protocols (PRISMA-P) 2015: elaboration and explanation. BMJ (Clinical Research Ed). 2015;350:g7647.

11. lankova I, Thompson-Leduc P, Kirson NY, Rice B, Hey J, Krause A, Schonfeld $S A$, DeBrase CR, Bozzette S, Schuetz P. Efficacy and safety of procalcitonin guidance in patients with suspected or confirmed sepsis: a systematic review and meta-analysis. Crit Care Med. 2018;46(5):691-8.

12. Roberts BW, Karagiannis P, Coletta M, Kilgannon JH, Chansky ME, Trzeciak S. Effects of $\mathrm{PaCO} 2$ derangements on clinical outcomes after cerebral injury: a systematic review. Resuscitation. 2015;91:32-41.

13. Hooijmans CR, Rovers MM, de Vries RBM, Leenaars M, Ritskes-Hoitinga M, Langendam MW. SYRCLE's risk of bias tool for animal studies. BMC Med Res Methodol. 2014;14:43.

14. Guyatt GH, Oxman AD, Vist GE, Kunz R, Falck-Ytter Y, Alonso-Coello P, Schunemann HJ, Group GW. GRADE: an emerging consensus on rating quality of evidence and strength of recommendations. BMJ. 2008; 336(7650):924-6.

15. Healthcare C, Utilization P. HCUP facts and figures. In: HCUP facts and figures, 2006: statistics on hospital-based care in the United States. Rockville: Agency for Healthcare Research and Quality (US); 2008.

16. Broccard AF, Hotchkiss JR, Vannay C, Markert M, Sauty A, Feihl F, Schaller MD. Protective effects of hypercapnic acidosis on ventilator-induced lung injury. Am J Respir Crit Care Med. 2001;164(5):802-6.

17. Laffey JG, Honan D, Hopkins N, Hyvelin JM, Boylan JF, McLoughlin P. Hypercapnic acidosis attenuates endotoxin-induced acute lung injury. Am J Respir Crit Care Med. 2004;169(1):46-56.

18. Laffey JG, Tanaka M, Engelberts D, Luo X, Yuan S, Tanswell AK, Post M, Lindsay T, Kavanagh BP. Therapeutic hypercapnia reduces pulmonary and systemic injury following in vivo lung reperfusion. Am J Respir Crit Care Med. 2000;162(6):2287-94.

19. Shibata K, Cregg N, Engelberts D, Takeuchi A, Fedorko L, Kavanagh BP. Hypercapnic acidosis may attenuate acute lung injury by inhibition of endogenous xanthine oxidase. Am J Respir Crit Care Med. 1998;158(5 Pt 1): 1578-84.

20. Sinclair SE, Kregenow DA, Lamm WJ, Starr IR, Chi EY, Hlastala MP. Hypercapnic acidosis is protective in an in vivo model of ventilator-induced lung injury. Am J Respir Crit Care Med. 2002;166(3):403-8.

Ready to submit your research? Choose BMC and benefit from:

- fast, convenient online submission

- thorough peer review by experienced researchers in your field

- rapid publication on acceptance

- support for research data, including large and complex data types

- gold Open Access which fosters wider collaboration and increased citations

- maximum visibility for your research: over $100 \mathrm{M}$ website views per year

At $\mathrm{BMC}$, research is always in progress.

Learn more biomedcentral.com/submissions 